\title{
Using air quality monitoring to reduce second-hand smoke exposure in homes: The AFRESH feasibility study
}

\author{
Ruaraidh Dobson' ${ }^{1}$ Rochel O'DonnelI', Marijn de Bruin ${ }^{1}$, Stephen Turner ${ }^{l}$, Sean Semple
}

\begin{abstract}
INTRODUCTION Few interventions to reduce second-hand smoke in homes where children are present have been successful. A novel intervention was developed that included personal airquality feedback. This study aimed to evaluate the feasibility and acceptability of delivering this theory-based intervention through small third-sector organisations in deprived areas within Scotland.

METHODS The setting was third-sector organisations in Scotland. Support workers used air quality monitors to give information on smoke-free homes to parents. This advice was structured around computer generated reports, co-developed with workers and target-group members. Participants received a monitor then received a report, which was discussed with a support worker. Two weeks later, the monitor was reinstalled and another report produced to evaluate success. Three participants and one support worker were interviewed afterwards to explore their experiences. RESULTS One centre out of six that were approached agreed to deliver the intervention. Four participants took part. All participants saw a decline in average concentrations of $\mathrm{PM}_{2.5}$ in their homes. In interviews, the participants and the support worker indicated that the intervention was acceptable and useful. The centres that declined to participate in the study cited a range of reasons, including a lack of staff time and perceived difficulties in recruiting members of the target population.

concLusions This intervention was acceptable for the target population tested, and may help participants to create smoke-free homes, although it is not possible to generalise the results of this small study. However, the resources required for the delivery of AFRESH do not match with the resources available in third-sector organisations, despite smoke-free homes being a policy priority.
\end{abstract}

\section{INTRODUCTION}

Second-hand smoke (SHS) exposure is a serious cause of ill health for children, contributing to illnesses such as meningitis, respiratory infections and sudden infant death syndrome. Worldwide, it is estimated that more than $40 \%$ of children are regularly exposed to second-hand smoke in the home ${ }^{1}$. Second-hand smoke can persist indoors at potentially harmful levels for long periods following even one cigarette ${ }^{2}$. Reducing this exposure is a public health challenge that has been embraced by policymakers and regulators ${ }^{3,4}$. However, there are few interventions that are effective in reducing SHS in deprived households with children ${ }^{5}$.

Fine particulate matter $\left(\mathrm{PM}_{2.5}\right)$ is a significant component of indoor air pollution, and has been widely used in tobacco control research as a marker for the presence of $\mathrm{SHS}^{6}$.

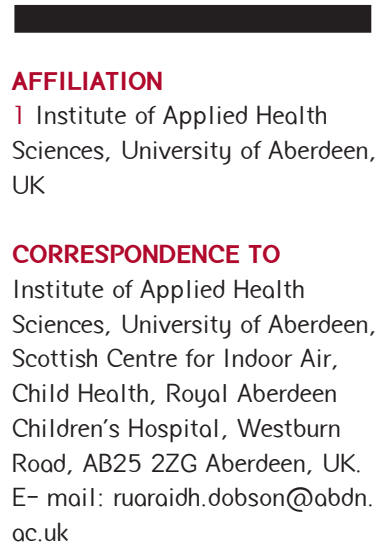

\section{KEYWORDS}

monitoring, second-hand smoke tobacco intervention, tobacco smoke pollution

Received: 27 January 2017 Revised: 5 April 2017

Accepted: 8 June 2017
Indoor air quality monitoring and feedback therefore has been explored as a potential motivator in interventions to encourage parents to keep smoke-free homes ${ }^{7-9}$. However, there is currently little evidence of interventions that have solid theoretical foundations in behaviour-change theory.

AFRESH (Finding Ways to Reduce Second-hand Smoke Exposure in Homes), a novel intervention to promote smokefree homes, was developed using intervention mapping ${ }^{10}$ to systematically develop a theory- and evidence-based behavioural intervention to reduce second-hand smoke in deprived households with children, with air quality feedback as a central component of the behavioural strategy. The full description of the intervention and its development process will be published separately. 
The aim of this study was to evaluate the feasibility and acceptability of delivering this theory-based intervention through small third-sector organisations in deprived areas within Scotland. While previous research in this area has relied on researchers providing air quality information to participants ${ }^{7}$, the present study gave full responsibility to nontechnical staff, thus testing the feasibility of the intervention in a real-world setting.

\section{METHODS}

\section{AFRESH intervention}

The AFRESH intervention is designed around the delivery of personalised air quality information to parents who do not live in smoke-free homes in deprived settings. The intervention is designed to be delivered by support workers employed by third-sector organisations, therefore widening the potential group of people who can deliver it by researchers and healthcare workers, as used in previous research ${ }^{7}$. The intervention is designed to be simple to deliver, to use a lowcost monitor and is structured around a series of modules and contacts between the worker and the parent: full details are available in the supplementary material ${ }^{11}$.

For this feasibility study, third-sector community centres were invited to take part, with their support workers receiving training on the intervention from the research team. Parents with a child under the age of six living in a smoking home were invited to participate in the intervention by a support worker. Following informed consent, the participant was given information about SHS by the thirdparty worker and provided with an air quality monitor to place in the home for approximately five days. Following the measurement period, the monitor was returned to the worker who then prepared an air quality report. Custom software $^{12}$ developed for AFRESH was used to facilitate the support worker in downloading and processing data, and in the preparation of air quality reports.

The report was given to the participant, and used as a focus to discuss planning for a smoke-free home with the worker. These discussions included techniques to allow exchange about the issue of SHS with other smokers and the available support to quit smoking entirely.

Two weeks later, the monitor was returned to the participant's home for another five-day period. A second report was produced including a comparison sheet (Figure 1). The worker and participant then discussed successes and challenges in keeping a smoke-free home.

\section{Ethics}

Ethical approval was provided by the College Ethics Review Board, College of Medicine and Life Sciences, University of Aberdeen.

\section{Figure 1. An example comparison report (showing test data), demonstrating changes in smoking behaviour over the course of the intervention}

Your average was:

\section{0}

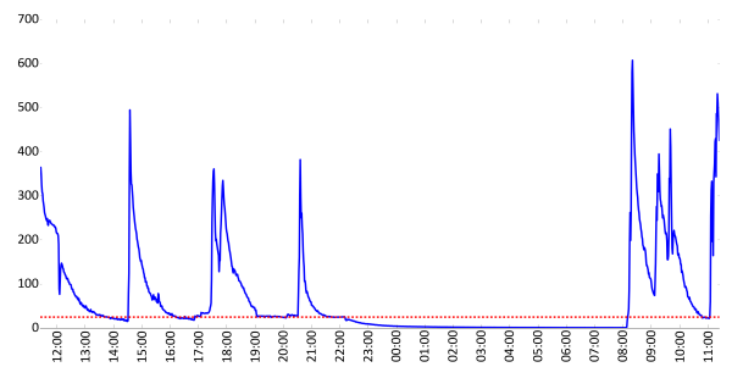

Your average is now:

15

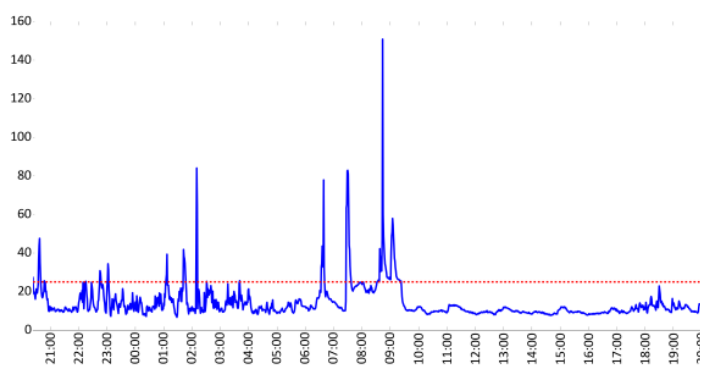

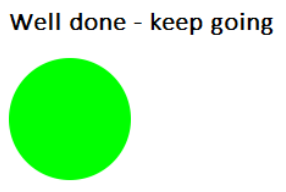

You reduced your levels
Your average is now:

\section{5}




\section{Recruitment}

A target of 20 intervention participants between two thirdsector centres was set at the beginning of the study, which was believed to be attainable based on previous work in this setting and in-line with the literature on good practice for feasibility studies testing intervention efficacy ${ }^{13}$.

Community centres were approached to take part through pre-existing relationships with research staff. These third-sector organisations employed workers in direct contact with members of the target population.

\section{Training}

Support workers taking part in the intervention were trained over two to three hour sessions. The first gave information about SHS and its effects on health and air quality, while the second gave information about the intervention. Following this session, an online course was developed to allow others to use the intervention ${ }^{14}$.

\section{Analysis}

The primary outcome measure for intervention efficacy was the change in average $\mathrm{PM}_{2.5}$ measured in each home following the intervention. Secondary outcome measures included self-reported changes in the rules of smoke-free home, including participant and staff experiences in using the intervention.

\section{Qualitative interviews}

Up to 10 parents were to be invited to take part in an individual telephone interview at the end of the intervention to explore; their experiences in taking part, and the impacts of the feedback they received on their smoking behaviour in the home. Support workers were also invited to take part in interviews at the end of the intervention, to explore their experiences during intervention delivery. These interviews used a semi-structured format.

\section{RESULTS}

\section{Recruitment}

One of the six centres invited to take part agreed to participate. In this centre, four participants were recruited and three of these participated in qualitative interviews. Five centres declined to take part citing reasons such as: staff turnover $(n=1)$, lack of staff time $(n=2)$, inability to recruit sufficient members of the target population $(n=2)$, and the perceived intrusiveness of the intervention $(n=1)$. One centre gave multiple reasons for declining to participate.

\section{Quantitative results}

Household average $\mathrm{PM}_{2.5}$ levels were lower in all four homes following the intervention, but the small sample size precluded statistical significance.

Baseline measurements lasted for durations between [5 days, 26 minutes] and [ 6 days, 6 hours, 22 minutes], while follow-up data were measured for durations between [3 days, 23 hours, 53 minutes] and [5 days, 7 hours, 15 minutes]. While the programme material suggested carrying out followup measurements two weeks after the end of the baseline measurement, in practice follow-up measurements began 18-39 days later (mean 28 days).

The mean measured $\mathrm{PM}_{2.5}$ was $80.5 \mu \mathrm{g} / \mathrm{m} 3$ (range 11-239 $\mu \mathrm{g} / \mathrm{m} 3$ ) at baseline and $66 \mu \mathrm{g} / \mathrm{m} 3$ (range $6-201 \mu \mathrm{g} / \mathrm{m} 3$ ) at follow-up. Reductions in average $\mathrm{PM}_{2.5}$ over this period ranged from 2-38 $\mu \mathrm{g} / \mathrm{m} 3$, but no home that had an average $\mathrm{PM}_{2.5}$ measured above the WHO guidance level at baseline had an average concentration below this value at follow-up.

\section{Qualitative results}

\section{Parent perceptions of the intervention}

Three of the four participants consented to take part in a semistructured telephone interview, conducted by a member of the research team, to discuss their experience of the intervention.

Changes to smoking behaviour were noted by two participants as a result of their personalised feedback; one had started smoking outside instead of indoors; a second was smoking inside, but now with the window open. The third had moved home between measurements, and was now smoking outside as smoking inside her new flat was prohibited as part of the rental agreement.

Parents stated that the intervention was acceptable, and found the air quality feedback reports understandable and meaningful:

"The graphs were quite understandable. It showed me the peak times [when SHS was highest]. It was very accurate too - I could see when I was smoking in the home and it was right"

When asked about benefits of taking part, all three commented on knowledge they had acquired through participating; for example:

"I've noticed that it [SHS] does linger longer than I thought it did. So when I come down in the morning, it's all clear [the air], but the machine says not."

"Now I know that it can make a difference to your kids if you don't smoke in the home. It's basically not normal to smoke around your children." 
"I've learnt that second-hand smoke is more harmful to kids than it is to adults. And that second hand smoke can cause asthma in children - I thought you were just born with it."

\section{Worker perceptions of the intervention}

The support workers were also interviewed about their experience of delivering the intervention. They valued the training received prior to conducting the intervention, and highlighted the importance of existing relationships with parents to aid recruitment. They spoke of capacity issues limiting recruitment given they were the sole worker on the project within their organization:

"we could have managed more[participants] if we had someone else doing it."

However, the staff member felt that the intervention itself was feasible and that feedback reports had the potential to change smoking behavior in the home, "especially with those that were high levels, because you could see that they were quite shocked with it, and it was interesting, just that graph and all the different colours and stuff. The colours really helped actually."

They considered that the intervention would be

"easy to integrate into our practice...I think the only thing would be the staff capacity."

\section{DISCUSSION}

\section{Recruitment of centres}

The main finding from this study was that recruiting centres to take part was problematic. The multi-step nature of the intervention was perceived to be excessive for reasons that included too great an impact on staff time. Carrying out one intervention requires five to seven contacts between a worker and a participant. This can involve travel to the participant's home or another location that potentially can take more time.

Even centres that initially expressed eagerness to take part were sometimes unable to do so, reflecting the high workload, limited resources and short-term funding cycles of the third-sector organisations approached. Expense could be another factor constraining the use of the intervention. The cost of purchasing the relatively inexpensive Dylos DC1700 can exceed $£ 400$ GBP (500 USD), a substantial sum for a small organisation. Staff time would represent an additional cost assuming an hour per contact paid at the median UK hourly wage ( $£ 11.78$ in 2015$)^{15}$, this would range from $£ 58.90$ - $£ 82.46$ per intervention. These costs would not apply where air quality monitoring equipment was already available, or in countries where labour costs are substantially lower than in Scotland.
The perceived inability to recruit smoking parents was unexpected since our previous experience was that many clients who were invited to take part and who attended the centres were regular smokers. Better-funded statutory bodies or recruiting participants directly may ameliorate these issues. The challenge in recruiting community "champions" is an important message for future studies and suggests that specific staff dedicated to smoke-free homes interventions are required rather than seeing this as an 'add-on' to the already high workload of support staff or support workers dealing with parents in deprived settings. Contacting potential partner organisations further in advance than the timeline of this study permitted, or providing them with financial compensation for engaging in the intervention programme, may improve participation rates.

\section{Feasibility of the intervention}

This feasibility study demonstrated that while the AFRESH intervention could be delivered by third-sector organisations working with individuals in low socioeconomic groups, the difficulties in doing so are substantial. Participants could understand, interpret and accept the results of air quality monitoring and, as with previous studies ${ }^{7}$, this may assist in encouraging changes to smoking behaviours.

All participants experienced reductions in household average $\mathrm{PM}_{2.5}$ concentrations by follow-up measurements though some changes were small. Carrying out the follow-up stage of the intervention repeatedly over a longer period may motivate participants to continue making changes, and provide evidence of the effectiveness of the different elements within the AFRESH programme.

\section{CONCLUSIONS}

Overall, those participants and workers who took part found the AFRESH programme acceptable and useful, but difficulties recruiting centres to take part would make the programme impossible to use widely in its current form. SHS levels fell modestly in all four homes, although the small sample size made statistical analysis of this decline impossible. Working with small community-based third-sector organisations presents practical and logistical challenges and these would be a significant barrier to using this model widely to promote smoke-free homes. Future research should focus on efforts to reduce the time and number of home visits or face-to-face contacts required to deliver the programme to those who express interest.

\section{REFERENCES}

1 GTSS Collaborative Group. A cross country comparison of exposure to secondhand smoke among youth. Tob Control 2006;15:ii4-19. doi:10.1136/tc.2006.015685 
2 Semple S, Latif N. How long does secondhand smoke remain in household air: analysis of PM2.5 data from smokers' homes. Nicotine Tob Res 2014;16:1365-70. doi:10.1093/ntr/ntu089

3 The Scottish Government. Creating a tobacco-free generation - a tobacco control strategy for Scotland. Edinburgh: 2013.

4 Mackay D, Haw S, Ayres JG, et al. Smoke-free Legislation and Hospitalizations for Childhood Asthma. N Engl J Med 2010;363:1139-45. doi:10.1056/NEJMoa1002861

5 Rosen LJ, Myers V, Winickoff JP, et al. Effectiveness of Interventions to Reduce Tobacco Smoke Pollution in Homes: A Systematic Review and Meta-Analysis. Int J Environ Res Public Health 2015;12:1604359 . doi:10.3390/ijerph121215038

6 Gorini G, Gasparrini A, Fondelli MC, et al. Second-Hand Smoke (SHS) Markers: Review of methods for monitoring exposure levels. Brussels: 2005.

7 Wilson I, Semple S, Mills LM, et al. REFRESH--reducing families' exposure to secondhand smoke in the home: a feasibility study. Tob Control 2013;22:e8.

doi:10.1136/tobaccocontrol-2011-050212

8 Klepeis NE, Hughes SC, Edwards RD, et al. Promoting smoke-free homes: a novel behavioral intervention using real-time audio-visual feedback on airborne particle levels. PLoS One 2013;8:e73251. doi:10.1371/journal.pone.0073251

9 Marsh J, McNeill A, Lewis S, et al. Protecting children from secondhand smoke: a mixed-methods feasibility study of a novel smoke-free home intervention. Pilot Feasibility Stud 2016;2:53. doi: 10.1186/s40814-016-0094-7

10 Bartholomew LK, Parcel GS, Kok G. Intervention mapping: a process for developing theory- and evidence-based health education programs. Health Educ Behav 1998;25:545-63.

doi: 10.1177/109019819802500502

11 Semple S, Apsley A, MacCalman L. An inexpensive particle monitor for smoker behaviour modification in homes. Tob Control 2013;22:295-8.

doi:10.1136/tobaccocontrol-2011-050401

12 Dobson R. AFRESH Software. 2015.Available at: http://bitbucket. org/ruaraidhd/dylosgui/ (accessed 8 June 2017).

13 Hertzog MA. Considerations in determining sample size for pilot studies. Res Nurs Health 2008;31:180-91.

doi:10.1002/nur.20247

14 ASH Scotland. AFRESH Training. 2016. Available at: http://afreshtraining.smokefreehomes.network/ (accessed 19 October 2016).

15 Office for National Statistics. Earnings and working hours. 2017. Availableat:https://www.ons.gov.uk/employmentandlabourmarket/ peopleinwork/earningsandworkinghours (accessed 17 March 2017).

ACKNOWLEDGEMENTS

This research was supported by a grant from the Medical Research Council's Public Health Intervention Development scheme.

The research team would like to thank Christine Foster and the staff and volunteers of Healthy Valleys, Lanarkshire, for their support in carrying out this work, and Beverley Scheepers and Joanne Buchan of ASH Scotland for their assistance in developing training material.

CONFLICT OF INTERESTS The authors have completed and submitted the ICMJE Form for Disclosure of Potential Conflicts of Interest and none were reported.

FUNDING

Medical Research Council PHIND Grant MR/ M026159/1

PROVENANCE AND PEER REVIEW

Not commissioned; externally peer reviewed 